\title{
Adherencia al Tratamiento de la Diabetes Mellitus Tipo 1, durante la Adolescencia. Una Perspectiva Psicológica
}

\author{
MARCO ARANEDA M. \\ 1. Psicólogo. Master en Psicología Clínica y de la Salud - Universidad de Bordeaux II. \\ Master en Psicología: Medicina Científica, Psicopatología y Psicoanálisis - Universidad de Paris VII.
}

\section{ABSTRACT \\ Treatment Compliance in Adolescents with Type 1 Diabetes Mellitus. A Psychological Perspective}

Introduction: A psychological perspective is offered for the evolution of the concept of "treatment compliance" among adolescents with Type 1 Diabetes Mellitus. Focus is placed on the main elements that have marked this evolution, with associated therapeutic modalities, from 1970 until now. Method: Literature review and discussion of significant papers. Conclusions: The concept of "treatment compliance" has evolved towards a dynamic multifactorial process, the result of an agreement between the patient and the treatment team, including a balance with factors necessary for the adolescent's development. In this light, various elements are reviewed that may be of importance to the treatment team.

(Key words: Diabetes, compliance, adolescence).

Rev Chil Pediatr 2009; 80 (6): 560-569

\section{RESUMEN}

Introducción: En este estudio se analizan la evolución del concepto de "adherencia al tratamiento" en la diabetes mellitus tipo 1, desde una perspectiva psicológica. Se enfoca en el período de la adolescencia y se revisan los principales elementos que han marcado la evolución de dicho concepto (y las prácticas terapéuticas que le están asociadas) desde la década 1970 hasta la actualidad. Método: Revisión bibliográfica, utilizando inicialmente la base de datos Elsevier - Science Direct y posteriormente a través de trabajos citados en la bibliografía encontrada inicialmente. Conclusión: El concepto de "adherencia al tratamiento" ha evolucionado hacia una dimensión dinámica, compuesta, multifactorial y fruto de un acuerdo entre el equipo médico y el paciente; además de la búsqueda de un equilibrio entre otros equilibrios necesarios para el desarrollo del adolescente. A la luz de estas constataciones, se discuten a lo largo de todo el artículo diversos elementos que el equipo de salud puede considerar en su acercamiento al adolescente diabético. (Palabras clave: Diabetes, adherencia, adolescencia).

Rev Chil Pediatr 2009; 80 (6): 560-569

Trabajo recibido el 22 de septiembre de 2008, devuelto para corregir el 22 de octubre de 2008, segunda versión el 18 de mayo de 2009, aceptado para publicación el 05 de agosto de 2009.

Correspondencia a:

Ps. Marco Araneda M.

E-mail: araneda.marco@gmail.com 


\section{Introducción}

Una vez diagnosticada la Diabetes Mellitus tipo 1 (DM1), el tratamiento se pondrá en marcha de forma urgente; en un comienzo llevado a cabo por el equipo de salud, pero rápidamente incorporando a la familia y al paciente en su ejecución ${ }^{1}$. Durante este período, se realizará una primera "educación terapéutica", que "tiene por objetivo formar al enfermo para que él pueda adquirir un saber-hacer adecuado, para llegar a un equilibrio entre su vida y el control óptimo de su enfermedad" (OMS, 1998)². La educación terapéutica estará destinada a enseñar una serie de procedimientos, hábitos y actitudes que buscan permitir al paciente reemplazar, por medio de su conducta consciente y voluntaria, una función biológica extremadamente compleja, que hasta el momento se realizaba en forma involuntaria y silenciosa.

Para el paciente, la toma de consciencia de que su cuerpo ha dejado de asegurar una función fundamental, y que es a través de una conducta voluntaria que él deberá asemejar esta función desaparecida, es un proceso extremadamente delicado desde el punto de vista psicológico. Un trabajo psíquico paralelo comienza también para los padres, quienes vivencian fuertes procesos emocionales en los días que siguen al diagnóstico ${ }^{3}$. Así, el trabajo psíquico que se impone tanto al niño como a sus padres, es de largo aliento; un trabajo que de ninguna manera se cumple en el breve plazo que va desde el diagnóstico hasta el comienzo de la educación terapéutica. Dicho de otra forma, hay un desfase entre la urgencia biológica que produce la detención de la producción de insulina y el tiempo psíquico que será necesario para incorporar la función de administración de insulina (y todos los otros componentes asociados del tratamiento) al espacio psíquico. Sin embargo, en un primer momento, la urgencia biológica es tal, que la colaboración esperada del paciente y la familia se transformará en un eje central del éxito del tratamiento. Es así como, principalmente desde la década de 1950, los investigadores se han esforzado por estudiar el concepto de "adherencia al tratamiento". Este concepto, junto con concertar hoy una gran cantidad de investigaciones, está experi- mentando cambios importantes gracias al conocimiento de las diversas dimensiones involucradas.

Nuestro objetivo es analizar la evolución del concepto de "adherencia al tratamiento" en la diabetes mellitus tipo 1 , desde una perspectiva psicológica, así como discutir distintos elementos de orden psicológico que pueden mejorar la comprensión y eficacia del equipo de salud en la atención del adolescente.

\section{Metodología}

La metodología incluyó una revisión en la base de datos Elsevier/Science Direct (términos "diabetes tipo 1", "adolescencia" y "adherencia al tratamiento" en francés, inglés y español) y posteriormente a través de trabajos citados en la bibliografía encontrada inicialmente. Se realizó posteriormente una selección de las fuentes, con acento en las publicaciones de revisión y de análisis crítico. Además, se consideró la experiencia clínica del autor como Psicólogo de la Unidad de Endocrinología de un hospital público pediátrico. Se consideró la adolescencia como "el período entre la infancia y la edad adulta... un período de transición durante el cual el niño cambia de manera física, mental y emocional para transformarse en adulto"4.

\section{Concepto de "adherencia" al tratamiento}

En medio siglo, diversos conceptos han sido creados para dar cuenta de este proceso. Los autores han optado por hablar de observancia, cumplimiento, adherencia o fidelidad terapéutica, sin que haya sido posible aún llegar a un consenso sobre la significación exacta y diferenciada de estos conceptos. En 1979, Haynes definía la observancia como "el grado de coincidencia entre el comportamiento del paciente (en término de toma de medicamentos, de seguimiento de regímenes alimenticios, de modificación del estilo de vida) y la opinión médica o la recomendación de salud que le ha sido prescrita"s. Esta definición, que pone el acento en una relación asimétrica (donde hay un sujeto que prescribe y otro que obedece) y centrada solamente en los aspectos técnicos del auto-cuidado del paciente, es hoy ampliamente cuestionada. 
Los investigadores han comenzado a percibir que hay otros factores que tienen una fuerte influencia en el proceso de "observancia", haciéndose necesaria una redefinición que permita una mejor comprensión y utilización de los resultados de estas investigaciones. Además, dicha modificación es imprescindible dadas las bajas tasas de adherencia observadas en pacientes con diversas patologías. En un estudio realizado con adolescentes chilenos, Ortiz reporta que aproximadamente el $50 \%$ de la muestra presenta un pobre control metabólico de su condición ${ }^{6}$. Resultado coincidente con los índices de adherencia encontrados por autores extranjeros en adolescentes con patologías crónicas ${ }^{7}$.

Además, contrariamente a la creencia general que indica que los problemas de adherencia son una especificidad de la etapa adolescente, hay diversos estudios que muestran que "de una forma general, los adolescentes con enfermedades crónicas se muestran en mayor dificultad que los niños, todo ocurre como si ellos no hicieran otra cosa que anunciar comportamientos finalmente adulto-morfos"8. Así, un $50 \%$ de pacientes adultos interrumpen un tratamiento por estatinas 6 meses después de su comienzo; mientras que la adherencia es de $20 \%$ o menos en pacientes re-hospitalizados por un segundo infarto. Y en el caso de adultos con Diabetes Mellitus tipo 2 se reportan tasas de observancia de 50\% ${ }^{9-11}$. Las dificultades de adherencia no son entonces una exclusividad ni de la DM1 ni de los adolescentes.

A partir de los hallazgos que se han realizado en torno a la comprensión del proceso de adherencia, una nueva definición ha sido propuesta por Hentinen (1988), quien considera la adherencia como "un proceso de cuidado activo y responsable, en el cual el individuo trabaja para mantener su salud en estrecha colaboración con el personal de salud"7. Esta definición coincide con las de otros investigadores ${ }^{7}$. Nos proponemos analizar a continuación los principales hallazgos que han llevado desde la definición de Haynes (1979) a la de Hentinen (1998).

\section{De la adherencia unitaria a la adherencia compuesta}

E1 tratamiento de la DM1 se desarrolla principalmente a través de: auto-controles de glice- mia (3 ó 4 al día), inyecciones de insulina (3 ó 4 al día), régimen alimenticio con cuantificación de hidratos de carbono, actividad física y asistencia a controles médicos periódicos. A pesar de que en la práctica clínica tiende a clasificarse a los pacientes ya sea en "adherentes" o en "no adherentes", las investigaciones muestran una gran variabilidad de la adherencia de un mismo paciente, dependiendo de qué componente se trate. Un paciente puede ser muy adherente a un componente y menos a otro. Una investigación realizada con adolescentes portadores de enfermedades crónicas encontró que solamente el $11 \%$ se mostraba adherente a los tres aspectos del tratamiento (toma de medicamentos, asistencia a los controles médicos y otras medidas terapéuticas) ${ }^{12}$. Resultado similar al encontrado en adolescentes diabéticos, donde se concluyó que no existía una correlación entre las adherencias a los 6 principales componentes del tratamiento ${ }^{8}$. La revisión de la literatura muestra además que los autores coinciden al reportar que de todos los elementos del tratamiento de la DM1, el régimen alimenticio es percibido por los pacientes diabéticos como el más difícil a respetar ${ }^{13}$; el mismo nivel de acuerdo existe respecto a que los adolescentes son, en general, mucho más adherentes a los elementos médicos del tratamiento (controles glicémicos e inyecciones de insulina) que a los elementos relacionados con los hábitos de vida (régimen alimenticio y actividad física).

Resulta importante entonces, al momento de detectar dificultades de adherencia, el evaluar la adherencia a cada uno de los componentes, estudiando sus causas y contexto, evitando la generalización y por sobre todo la rotulación del paciente como "no-adherente".

\section{De la adherencia como dicotomía a la adherencia como dimensión}

No hay sólo dos estados posibles para este fenómeno: "adherencia" y "no-adherencia", es un continuo "que va desde la fidelidad ejemplar a las prescripciones y programas terapéuticos hasta los rechazos completos de los tratamientos o los abandonos del seguimiento médico"s. Entre ambos extremos, una multitud de estados intermedios muestran la mayor o menor ambivalencia con la cual el paciente se enfrentará 
cotidianamente al plan terapéutico. Ser receptivo a estos estados intermedios (que constituyen una rica fuente de información sobre la forma como el paciente está vivenciando su tratamiento) permitirá prevenir el desarrollo de rechazos masivos del mismo; actuando a tiempo para la mantención del tratamiento y de la relación de confianza en la que éste se apoya.

\section{De la adherencia fija a la adherencia dinámica}

La adherencia además fluctúa en el tiempo, es dinámica ${ }^{14}$, reflejo del dinamismo de los factores que influencian el nivel de adherencia de cada paciente (factores que analizaremos más adelante). A modo de ejemplo, un paciente de 12 años de edad, que hasta el momento no ha manifestado mayor inconveniente en privarse de comer determinado tipo de alimentos, podría manifestar una variación de dicha adherencia, durante las fiestas con sus pares, donde consumir dicho alimento puede ser visto como una forma de mostrar que se pertenece al grupo.

\section{De la adherencia única a la adherencia que forma parte de un conjunto de adherencias.}

El equilibrio glicémico no es el único equilibrio del cual un adolescente debe ocuparse. Él debe también encontrar: a) un equilibrio en la percepción de su cuerpo (que cambia progresivamente hacia la forma adulta); b) un equilibrio en la imagen sobre sí mismo, sus padres, sus pares y su comunidad (imágenes que cambian aceleradamente durante la adolescencia); c) un equilibrio entre la dependencia que aún existe respecto de su familia (emocional, económica, normativa) y la autonomía que se abre frente a él como una posibilidad atractiva e incierta.

El cuidado del equilibrio glicémico es una tarea más que viene a sumarse a las tareas normales del desarrollo. Es fundamental que el equipo de salud pueda tener en perspectiva estos equilibrios múltiples a la hora de detectar dificultades de adherencia en un adolescente ${ }^{15}$; ya que muchas veces los desequilibrios encontrados en el control glicémico pueden estar relacionados con opciones que el adolescente ha debido tomar para no perder el equilibrio en otras áreas de su vida ${ }^{16}$. Shaw (2001), reporta que "los adolescentes y sus familias están constantemente intentando balancear las necesidades del cuidado de su salud con las necesidades de una vida normal" 17 .

Uno de los grandes problemas que se presentan al evaluar dificultades de adherencia, es que pueden corresponder a elecciones forzadas y dolorosas que las personas realizan precisamente para no perder el equilibrio (del desarrollo, del psiquismo). En la gran mayoría de los casos, los pacientes y sus familias han comprendido bastante bien las indicaciones desde las primeras educaciones terapéuticas. Será una tarea de todo el equipo el estar atentos a percibir estas elecciones dinámicas que se esconden bajo una conducta de "no-adherencia", sin reducir jamás la adherencia a un simple problema de educación terapéutica ${ }^{18}$.

Curiosamente, las elecciones forzadas no se asocian solamente a las situaciones de "noadherencia", sino que también están presentes bajo las conductas de adherencia. Grey et al (1998), señalan: "Incluso los adolescentes que alcanzan con éxito las metas de hemoglobina glicosilada de la terapia pueden percibir la "diabetes" como algo que tiene un impacto negativo en sus vidas, estar deprimidos y encontrar que el manejo de la diabetes es difícil. Los equipos que tratan la diabetes necesitan prestar igual atención a las necesidades psico-sociales del adolescente juicioso y no-rebelde, con una diabetes bien controlada y con una familia que lo apoya, así como lo hacen con las necesidades del adolescente rebelde con una diabetes pobremente controlada" 19 . Normo-glicemia no es entonces necesariamente sinónimo de bienestar psicológico. Esto no significa que para lograr este equilibrio en la calidad de vida sea necesario abandonar las metas terapéuticas del control glicémico. Los adolescentes no esperan ni desean esto, desean un equipo de salud atento a sus necesidades médicas, que pueda darle indicaciones claras sobre cómo cuidar su salud y que no se desanime frente a la primera oposición que él manifieste. Pero ellos esperan además, que el equipo de salud pueda escuchar empáticamente: a) el relato de las dificultades que se presentan en la vida cotidiana al ejecutar las medidas terapéuticas; b) que el equilibrio glicémico es uno más de una lista de equilibrios 
múltiples y simultáneos, y c) los sentimientos de desánimo, ansiedad, disconformidad, miedo, rabia, logro y cambio que los pacientes experimentan frente a la diabetes. El poder hablar de esto, que sea reconocido por el equipo de salud y recibir una respuesta comprensiva y reflexionada para su situación particular, permite a los adolescentes ganar en comprensión y aceptación de sí mismos, conocer mejor a quienes se ocupan de su salud (aprendiendo cómo reaccionan frente a su oposición o dificultades) y validar el espacio terapéutico como un espacio donde la comunicación y la palabra tienen un valor humanizante.

Además, el equilibrio metabólico no depende sólo de la correcta aplicación de las indicaciones terapéuticas, ya que existen factores asociados al cambio hormonal de la pubertad que tienen repercusiones sobre los niveles glicémicos. Esto podría provocar algunas atribuciones erróneas sobre la "responsabilidad" de determinada variación en los indicadores de control. Junto a esto, el temor a los episodios de hipoglicemia, secundario a descompensaciones importantes, puede cronificarse y dificultar la adherencia. Muchos pacientes (adolescentes y adultos) son guiados por el temor a los episodios de hipoglicemia ${ }^{20,21}$. Ellos podrían intentar mantenerse preventivamente en un cierto nivel de hiperglicemia, en la creencia que esto los podrá mantener a salvo de sufrir una nueva hipoglicemia (en vez de atenerse al rango indicado por el médico). Independientemente de que el rango indicado por el médico no aumente el riesgo de una hipoglicemia el paciente podría percibir dicho rango como demasiado justo $\mathrm{y}$ que lo deja "al borde de hipoglicemias", él intentará evidentemente modificar dicho rango, muchas veces sin consultar la opinión médica. Esta conducta suele asociarse a fuertes sentimientos de culpabilidad y de temor por las complicaciones secundarias que su decisión podría implicar ${ }^{20}$. Esto muestra como las decisiones tomadas por el paciente se realizan siempre en un encuadre complejo de equilibrios múltiples.

\section{De la adherencia a una orden a la adherencia a un acuerdo}

El ser humano adhiere más a lo que ha elegido que a lo que le ha sido impuesto. La definición de Hentinen (1998) pone el acento en la colaboración mutua. Los estudios actuales muestran que los adolescentes (y los adultos también) adhieren en mayor medida cuando han podido participar en la discusión del plan terapéutico e influir en el ajuste de dicho plan a su propia situación personal. Cuando la enfermedad ha comenzado durante la infancia, son los padres quienes se encargan de administrar y controlar cotidianamente el tratamiento del niño. Sin embargo, esta situación cambia durante la adolescencia temprana, cuando los pacientes deben comenzar a responsabilizarse por aspectos de los cuales antes respondían sus padres. Para el médico comienza a haber un cambio de interlocutor, de los padres al adolescente $^{22}$.

En este contexto, "para un médico, puede ser difícil no interpretar una no-observancia en términos de traición de la confianza o, más simplemente, de no respeto de la autoridad" " la gran mayoría de las veces no se trata de una desobediencia, sino de un intento del adolescente por ganar ingerencia en algo que le incumbe profunda y vitalmente, un intento por tomar la palabra y participar activamente. Se produce entonces una situación paradojal porque su conducta de no-adherencia no corresponde a una conducta de pasividad sino a un deseo importante por participar y decidir. Por lo demás, esta autonomización constituye uno de los objetivos claves del auto-cuidado del paciente. Para el paciente, será necesaria una coherencia en esta autonomía, la cuál deberá respetarse no solamente en la ejecución de las conductas del tratamiento, sino también en la definición de las mismas. De hecho, en los protocolos actuales que incluyen insulina de acción rápida los pacientes pueden regular sus dosis de insulina hasta cierto grado, dependiendo de la dieta, ejercicio, condición física, etc ${ }^{13}$. En complemento, la posibilidad de tener un margen de control en el manejo del tratamiento se asocia a menudo con un mayor bienestar psicológico. Finalmente, una comunicación fluida, en donde ambos participantes pueden opinar y negociar lo que es negociable en la fijación de las metas terapéuticas, es fundamental y benéfica para la adherencia ${ }^{9,16,20,23,24}$. La re- 
lación médico-paciente es una relación de dos expertos, un médico experto en el sistema endocrinológico y un adolescente experto en su propia vida ${ }^{24}$.

El adolescente no se dedica a cuestionar la autoridad de los adultos por un impulso sin sentido. Él busca de diversas maneras comenzar a participar en el delineamiento de sus proyectos y de su vida, adquirir la experiencia de la toma de decisiones. Por lo demás, el logro de la autonomía le será exigido más tarde por la sociedad a la que pertenece. Ya que esta autonomía no se logra de un momento a otro, será importante favorecer el tiempo de la adolescencia como un período en el cuál se podrán ofrecer al adolescente espacios donde podrá participar progresivamente (y no de forma abrupta) en la toma de decisiones, sintiendo siempre que hay adultos (padres, equipo de salud) que estarán presentes, que no dudarán en anticiparse a situaciones complejas y que le señalarán claramente: a) qué aspectos de la realidad le permiten una elección; b) qué aspectos de la realidad no quedan aún a su elección y; c) qué aspectos no son elegibles, sino que son impuestos por la realidad (de su cuerpo, de su sociedad).

\section{De la adherencia como proceso voluntario a la adherencia como proceso multifactorial}

Muchas de las percepciones respecto de los adolescentes o las familias que no cumplen con las indicaciones médicas, están basadas en la suposición inconsciente que la conducta de "noadherencia" es una conducta voluntaria del paciente, que depende sólo de sí mismo, de su nivel de comprensión de la importancia del tratamiento y de su "buen juicio". Sin embargo, uno de los mayores hallazgos de la investigación sobre la adherencia, es el carácter multifactorial de ésta. Diversos investigadores han descubierto una serie de factores que presentan lazos estadísticamente significativos con la adherencia. Aunque revisar en detalle dichos factores sobrepasa los límites del presente artículo, reseñaremos a continuación algunos de ellos:

a) Factores ligados a la enfermedad y al tratamiento: La complejidad del régimen tera- péutico, su duración, la incomodidad física que produce, los efectos secundarios indeseables y la necesidad de un cambio en los hábitos de vida son factores relevantes a la hora de evaluar el grado de adherencia del paciente ${ }^{5}$. En un estudio realizado con adolescentes diabéticos, Castro (1991) encontró que la complejidad del tratamiento "era el problema mayor en la mantención de una buena adhesión, para el 90\% de los adolescentes. A pesar de tener un conocimiento correcto del tratamiento, los niños crean una representación mental de lo que el tratamiento debería ser y se vuelven muy adherentes a su propio esquema subjetivo" 25 . Este estudio instala dos temas importantes para la práctica clínica: a) cómo cada paciente se representa cognitiva y afectivamente el tratamiento, y b) lo imprescindible de incorporar al paciente en la discusión, antes de la fijación de las metas terapéuticas (de esta forma, el médico estará en conocimiento de las dificultades percibidas por el paciente y podrá llegar a un acuerdo sobre las modificaciones que sean pertinentes).

b) Especificidad de la adolescencia: A medida que un niño con DM1 avanza hacia la adolescencia, los procesos de negociación por la toma de decisiones (con sus padres y con el equipo de salud) van en aumento ${ }^{5}$. A nivel inconsciente, para un adolescente cuya diabetes comenzó durante la infancia, el seguimiento de las conductas de autocuidado puede comenzar a tomar la significación de aceptar el rol de "niño" o de "niño enfermo". Ambos roles entran en oposición con su necesidad de ganar autonomía y de transformarse en adulto. Es por esto que el equipo de salud deberá ser especialmente sensible a la significación que toma, para cada adolescente en particular, el seguimiento de las metas terapéuticas.

Existen además factores del desarrollo cognitivo y emocional que es importante considerar. Diversos autores han mostrado que los adolescentes más jóvenes usan aún un pensamiento concreto en la toma de decisiones, y que incluso cuando el pensamiento más abstracto se encuentra en desarrollo, éste deja el lugar al pensamiento concreto en las situaciones cargadas emocionalmente. Los más jóvenes perciben además un rango más estrecho de solucio- 
nes posibles a un problema, tienen poca tendencia a considerar las consecuencias de largo plazo y se apresuran en la toma de decisiones para acomodarse a las opiniones de grupos importantes para ellos ${ }^{17}$. Un ejemplo de esto: los adolescentes con DM1 perciben un mayor riesgo de desarrollar complicaciones a corto que a largo plazo y se perciben a sí mismos en menor riesgo que sus pares diabéticos ${ }^{26}$. Otro argumento más para comprender que frente a un problema de adherencia, el poner el acento en las complicaciones a largo plazo puede ser inefectivo (por la dificultad cognitiva a representarse el futuro lejano) y contraproducente (por el bloqueo afectivo que puede producir la perspectiva de complicaciones difíciles a aceptar psicológicamente). Es importante además considerar que la tarea fundamental de todo adolescente es la construcción de una identidad que le permita la continuación de su desarrollo adulto. Para ello deberá atravesar una crisis de cuestionamiento de sus objetivos y valores (Marcia, 1980) ${ }^{4}$.

c) Relación con los pares: A medida que se distancia progresivamente de los padres, el adolescente se acerca al grupo de pares. La necesidad de asemejarse a sus pares (normalización) y evitar toda característica que lo pueda hacer diferente de ellos será fundamental. Dependiendo de la actitud del grupo de pares, un adolescente manejará muchas veces su tratamiento en relación a esta ecuación de normalización. Hay grupos de pares que adaptan sus actividades a las necesidades del tratamiento del adolescente diabético, con aceptación y empatía, favoreciendo en él el sentimiento de ser apoyado; lo cual se asocia a su vez a una mejor adherencia al tratamiento ${ }^{5}$. Mientras que cuando el tratamiento implica un cambio en los hábitos de vida, que lo diferencia demasiado de los pares, la adherencia puede verse comprometida $^{24,27}$. Sin embargo, un apoyo demasiado intenso puede producir el efecto inverso en la adherencia, provocando una sensación de estrés y agobio ${ }^{5}$.

d) Factores familiares: La percepción que los padres tienen respecto del equipo de salud, el tratamiento y su eficacia, son fundamentales a la hora de prevenir las dificultades de adherencia. Los padres pueden tener percepciones que van desde una confianza ciega en el equipo de salud a una actitud de temor de ser reemplazados en su rol parental de toma de decisiones respecto a su hijo ${ }^{5}$. Un clima familiar positivo, relaciones abiertas entre los miembros de la familia, y una expectativa positiva de los padres respecto a la eficacia del tratamiento ${ }^{5}$, se encuentran asociados a una mejor adherencia.

Otro factor importante es la percepción que los padres tienen de la autonomía posible de sus hijos. Un interesante estudio realizado con 127 adolescentes con DM1 y sus madres, señala que puede ser tremendamente problemático que las madres varíen su grado de involucramiento en el control del tratamiento guiándose por los signos físicos de desarrollo de su hijo (pubertad) más que en la evaluación del grado real de autonomía y competencia requerida para autocontrolar el tratamiento ${ }^{28}$. Este estudio, plantea también, la importante pregunta respecto a los criterios utilizados para el traspaso de los pacientes desde los hospitales pediátricos a los hospitales de adultos.

\section{e) Factores relacionados con el equipo} de salud: En un estudio con adolescentes epilépticos, se encontró que las principales razones de insatisfacción con la atención de salud son: un tiempo de espera excesivo, médicos diferentes en vez de un interlocutor único y la ausencia de claridad en la comunicación médico-paciente ${ }^{5}$. Mientras que un estudio japonésfinlandés muestra que una mejor adherencia está relacionada con el apoyo que los adolescentes sienten que reciben de los médicos, enfermeras y padres ${ }^{23}$. Estos autores señalan que una parte importante de los adolescentes tienen la sensación que el equipo de salud fija las metas terapéuticas sin explicarles los objetivos y que se interesa más por la enfermedad que por el paciente. Olsen y Sutton (1998) observaron en una muestra de adolescentes con DM1, la importancia que los pacientes atribuyen no sólo al apoyo informativo sino también al apoyo afectivo (dar confianza al paciente) ${ }^{29}$. La confianza y el bienestar resultantes se asocian a un mejor control glicémico y adherencia. Otros autores consideran la adherencia como la re- 
sultante de intercambios e influencias entre y dentro de los siguientes subsistemas: cuidador (padres)-equipo médico, niño-equipo médico, cuidador-niño ${ }^{30}$.

f) Factores personales: Uno de los mayores hallazgos de la investigación es que las percepciones subjetivas de las personas tienen mayor peso predictivo sobre la adherencia que los factores objetivos. Es por esto que el punto central de la evaluación será captar cómo cada adolescente y familia perciben: la enfermedad, cada uno de los componentes del tratamiento, el futuro y sus propias capacidades para hacer frente al mismo. Las razones que explican esta variabilidad en la percepción de una realidad supuestamente objetiva son múltiples y actúan de forma inconsciente. La adherencia será finalmente el resultado visible de una serie de procesos complejos, que toman un ordenamiento dinámico y particular en cada adolescente y familia.

Así por ejemplo, los pacientes que creen que su salud depende de factores externos (Externalidad) (especialmente la "suerte") presentan un peor control metabólico, mientras que aquellos que creen que la salud depende de factores internos (Internalidad) presentan un buen control glicémico, estabilidad ponderal y bienestar psicológico ${ }^{31}$. Más específicamente, dentro de la Internalidad existirían dos subcategorías: la autonomía (asociada a un buen ajuste a la diabetes) y la auto-culpabilización (asociada a un mal ajuste posterior a la enfermedad) ${ }^{32}$. Por otro lado, un estudio realizado con adolescentes diabéticos muestra que quienes tenían un conocimiento demasiado detallado sobre la enfermedad tenían dificultades de adherencia $^{33}$. En la misma línea, una información demasiado concentrada entregada por el equipo de salud puede algunas veces tener un efecto nocivo sobre los pacientes que no tienen la tendencia (ni el deseo) de buscar información detallada sobre las patologías que pade$\operatorname{cen}^{31}$. Una de las tareas del equipo de salud será entonces evaluar el justo equilibrio entre lo que es imprescindible y necesario informar y lo que el paciente y la familia están en condiciones de escuchar y tolerar psicológicamente en cada momento.
El Modelo de Creencias en Salud (Health Belief Model) ha permitido constatar que cuando los beneficios percibidos del tratamiento son mayores que los costos (dificultades, restricciones, etc) el sujeto manifiesta una adherencia elevada ${ }^{31}$. Además, es importante destacar que a pesar que hemos hablado aquí de "factores personales" esto no significa en ningún caso que ellos dependan exclusivamente de la voluntad del adolescente. Muchas veces, estos procesos, a pesar de ser localizables en el paciente, dependen a su vez de los otros factores (familiares, del equipo de salud, de la enfermedad, etc), los cuales influyen en la forma como el adolescente percibe su propia situación.

\section{Conclusión}

El concepto de adherencia ha evolucionado desde un concepto centrado en una relación asimétrica de indicación-obediencia (1979) a una relación de colaboración médico-paciente-familia (1998). Una cantidad cada vez mayor de investigaciones consideran a la adherencia como una dimensión dinámica, compuesta, multifactorial y de acomodación mutua. La cual es, a su vez, una más de un conjunto de adherencias del adolescente.

En vez de considerarla como un problema a remediar rápidamente, los autores invitan a incorporar la "evaluación de la adherencia" como un ítem más de la conversación de todo médico con su paciente adolescente; un tema abierto, del cuál se pueda hablar sin el temor de ser catalogado abruptamente de "irresponsable". De hecho, los adolescentes se sienten aliviados de poder hablar con su médico de sus dificultades para adaptarse al tratamiento y para equilibrar éste con los otros aspectos de su vida ${ }^{18,24,34}$; particularmente con los desafíos que los cambios físicos y emocionales de la adolescencia plantean. Establecer una comunicación abierta y serena como eje central y permanente de la relación ${ }^{35}$ permitirá no sólo tratar efectivamente las dificultades de adherencia que pudieran poner en riesgo el bienestar del paciente, sino también prevenirlas en una proporción muy importante. 
Finalmente, el equilibrio en el tratamiento de la diabetes es un desafío. Esto se debe no sólo a razones biológicas (de ajuste de dosis de insulina) o conductuales, sino también a que un equilibrio durable deberá enfocarse en los intentos del adolescente y su familia por encontrar un sentido a la enfermedad y al futuro que ellos desean para sí mismos. La no-adherencia al tratamiento es una señal para "restaurar el sentimiento de control del niño, el sentirse respetado, que su palabra es tomada en cuenta; la importancia también de reconocer su ambivalencia: su deseo de sanar tanto como su sensación de desamparo y rabia" ${ }^{36}$. La no-adherencia constituye entonces una señal en el trabajo de cuidado de la salud que los equipos médicos realizan.

\section{Referencias}

1.- Gillet P, Beaufrère B, Asenjo-Ramis S, François R: La vie quotidienne du jeune diabétique insulinoprivé. Insertion scolaire, sociale et professionnelle. Ann Pédiatr. (Paris) 1986; 33 (10): 927-34.

2.- Baudier F, Leboube $G$ : Éducation thérapeutique du patient et disease management: pour une $3^{\mathrm{e}}$ voie "à la française"? Santé publique 2007; 4 (19): 335-40.

3.- Ricard-Malivoir S: Remaniements psychiques observés chez des parents lors de l'annonce du diagnostic de diabète insulino-dépendant chez leur enfant. Diabetes \& Metabolism (Paris) 1997; 23: 171-3.

4.- Bee H, Boyd D: Psychologie du développement. Les âges de la vie. Bruxelles: De Boeck éditeurs, 2003.

5.- White-Koning M, Bertozzi-Salamon AI, Vignes M, Arnaud C: L'observance des adolescents atteints de cancer. Bull Cancer 2007; 94 (4): 349-56.

6.- Ortiz M, Ortiz E: Adherencia al tratamiento en adolescentes diabéticos tipo 1 chilenos: una aproximación psicológica. Rev Méd Chile 2005; 133: 307-13.

7.- Hentinen M, Kyngäs H: Diabetic adolescents' compliance with health regimes and associated factors. Int J Nurs Stud 1996; 33 (3): 325-37.

8.- Alvin P: L'adolescent et l'observance au traitement, J Pédiatr Puericulture 2000; 13: 225-9.

9.- Gallois P, Vallée JP, Le Noc Y: L'observance des prescriptions médicales: quels sont les facteurs en cause? Comment l'améliorer? Médecine 2006; 402-6.

10.- Golay A, Nguyen Howles M, Mateiciuc S, et al: Améliorer l'observance médicamenteuse. Med Hyg 2004; 62: 909-13.
11.- Osterberg L, Blaschke T: Adherence to medication. N Engl J Med 2005; 353 (5): 487-97.

12.- Michaud PA, Frappier JY, Pless IB: La compliance d'adolescents souffrant d'une maladie chronique. Arch Fr Pediatr 1991; 48: 329-36.

13.- Haugbolle L, Devantier K, Frydenlund B: A user perspective on type 1 diabetes: sense of illness, search for freedom and the role of the pharmacy. Patient Education and Counseling 2002; 47: 361-8.

14.- Fielding D, Duff A: Compliance with treatment protocols: interventions for children with chronic illness. Arch Dis Child 1999; 80: 196-200.

15.- Basdevant A, Le Barzic M, Pouillon M, Guy-Grand $B$ : Le diabète de l'adolescent. Le point de vue du diabétologue d'adultes. La Presse Médicale 1985; 14 (1): 13-5.

16.- Spitz E: Les stratégies d'adaptation face à la maladie chronique. En: Fischer GN (sous la direction de). Traité de Psychologie de la santé, Paris: Dunod 2002; 261 82.

17.- Shaw R: Treatment adherence in adolescents: development and psychopathology. Clinical Child Psychol and Psychiatr 2001; 6 (1): 137-50.

18.- Alvin P, Rey C, Frappier JY: Compliance thérapeutique chez l'adolescent malade chronique. Arch Pédiatr 1995; 2: 874-82.

19.- Grey M, Boland E, Yu Ch, Sullivan-Bolyai S, Tamborlane $W$ : Personal and Family Factors associated with quality of life in adolescents with diabetes. Diabetes Care 1998; 21 (6): 909-14.

20.- Kyngäs H, Kroll T, Duffy M: Compliance in adolescents with chronic diseases: a review. J Adolesc Health 2000; 26: $379-88$.

21.- Cox D, Irvine A, Gonder-Frederick L, et al: Fear of hypoglycemia: quantification, validation and utilization. Diabetes Care 1987; 10 (5): 617-21.

22.- Kyngäs $H$ : A theoretical model of compliance in young diabetics. J Clin Nurs 1999; 8: 73-80.

23.- Kyngäs H, Rissanen M: Support as a crucial predictor of good compliance of adolescents with a chronic disease. J Clin Nurs 2001; 10: 767-74.

24.- Kyngäs H, Hentinen M, Barlow J: Adolescents' perceptions of physicians, nurses, parents and friends: help or hindrance in compliance with diabetes selfcare? J Adv Nurs 1998; 27: 760-9.

25.- Castro D: Aspects psychologiques de la compliance au traitement chez l'enfant diabétique insulinodépendant. Ann Pédiatr (Paris) 1991; 38 (7): 455-8.

26.- Patino AM, Sánchez J, Eidson M, Delamater A: Health beliefs and regimen adherence in minority adolescents 
with type 1 diabetes. J Pediatr Psychol 2005; 30 (6): 503-12.

27.- Seiffge-Krenke I: Problèmes de santé et stratégies de coping à l'adolescence. En: Tap P., Malewska-Peyre (sous la direction de). Marginalités et troubles de la socialisation, Paris: PUF, 1993; 133-52.

28.- Palmer D, et al: The role of autonomy and pubertal status in understanding age differences in maternal involvement in diabetes responsibility across adolescence. J Pediatr Psychol 2004; 29 (1): 35-46.

29.- Olsen R, Sutton J: More hassle, more alone: adolescents with diabetes and the role of formal and informal support. Child: Care, Health and Development 1998; 24 (1): 31-9.

30.- De Civita M, Dobkin P: Pediatric adherence as a multidimensional and dynamic construct, involving a triadic partnership. Journal of pediatric psychology. 2004; 29 (3): 157-69.

31.- Chamberlain K, Quintard B: Les déterminants psychosociaux de l'ajustement au diabète. En:
Bruchon-Schweitzer M., Quintard B. (sous la direction de). Personnalité et maladies. Stress, coping et ajustement, Paris: Dunod, 2001; 93-112.

32.- Peyrot M, Rubin R: Structure and correlates of diabetes-specific locus of control. Diabetes Care 1994; 17 (9): 994-1001.

33.- Hamburg B, Inoff G: Relationships between behavioral factors and diabetic control in children and adolescents: a camp study. Psychosomatic Medicine 1982; 44 (4): 321-39.

34.- Oppenheim D, Dauchy S, Hartmann O: Refuser le traitement: responsabilité et autonomie du patient. Bull Cancer 2006; 93 (1): 133-7.

35.- Alvin P: La non-compliance thérapeutique: vérité pénible à dire ou difficile à entendre? Arch Pédiatr 1997; 4: 395-7.

36.- Oppenheim D: Comment aborder les problèmes de non-compliance en pédiatrie? L'expérience de 1'oncologie pédiatrique. Neuropsychiatr. Enfance Adolesc 1996; 44 (6-7): 248-57. 